\title{
Ventricular arrhythmias in chronic Chagas cardiomyopathy: Can studying myocardial sympathetic denervation provide the answers?
}

\author{
Vineet Kumar, $M D^{a}$ \\ ${ }^{a}$ Division of Cardiovascular Diseases, University of Alabama at Birmingham, Birmingham, AL
}

Received Jun 7, 2016; accepted Jun 8, 2016

doi:10.1007/s12350-016-0587-z

\section{See related article, pp. 75-83}

Carlos Chagas in the beginning of 20th century described a disease entity affecting millions of people in Latin America caused by the parasite Trypanosoma cruzi. $^{1}$ Cardiac involvement was and remains the most serious form of this disease and is responsible for majority of cases of nonischemic cardiomyopathy in Latin America. With globalization and increase in international travel over the last few decades, this entity is no longer confined to Latin America and is being increasingly diagnosed in other geographic areas around the world. ${ }^{2}$ Ventricular arrhythmias (VA) are a key feature of chronic Chagas cardiomyopathy (CCC) with a high incidence of sudden cardiac death in this patient population. Arrhythmias can occur in individuals with normal or near normal left ventricular systolic func$\operatorname{tion}^{2,3}$ and identification of individuals at high risk of arrhythmic event remains a challenge.

\section{CARDIAC DENERVATION AND CHAGAS DISEASE}

Cardiac denervation plays an important role in arrhythmogenesis in different cardiomyopathies and its importance in risk stratification of patients is a subject of intense interest. Fritz Koberle studied autopsy specimens of patients with Chagas disease several decades ago and demonstrated significant reduction in cardiac

Reprint requests: Vineet Kumar, MD, Division of Cardiovascular Diseases, University of Alabama at Birmingham, 930 Faculty Office Towers, 510 20th Street South, Birmingham, AL 35294-0006; vkumar@uab.edu

J Nucl Cardiol 2018;25:84-5.

$1071-3581 / \$ 34.00$

Copyright (c) 2016 American Society of Nuclear Cardiology. intramural neuronal population compromised mostly of parasympathetic neurons. ${ }^{4}$ Although this finding can be seen in other cardiomyopathies, its severity was more profound in patients with CCC.,6 Parasympathetic neuronal loss is thought to occur during the acute phase of the disease, by combination of factors, including, direct damage to the nerve terminals by the parasite, intense perineuronal inflammatory reaction, and immunological mechanisms. ${ }^{7}$ Most early evidence was for parasympathetic denervation, and it was proposed that Chagas cardiomyopathy was a result of chronic sympathetic overstimulation; however, subsequent studies refuted this hypothesis by showing the presence of sympathetic denervation in these patients. ${ }^{8}$ Cardiac sympathetic denervation can be studied using radiolabelled norepinephrine (NE) analogs with single-photon emission computed tomography (SPECT) or positron emission tomography (PET) cameras. Metaiodobenzylguanidine (mIBG) labeled with radioactive iodine is the most commonly used analog and is easily imaged with SPECT camera. $\mathrm{C}^{11}$-labeled hydroxyephedrine (HED) is the most commonly studied PET agent. It has higher selectivity for neuronal NE uptake mechanism and more for homogenous uptake which may allow better differentiation of denervated myocardium from normal innervation. ${ }^{9}$

In the present study, Gadioli et al studied sympathetic denervation and resting myocardial perfusion using $\mathrm{mIBG}$ and $\mathrm{Tc}^{99}$-sestamibi SPECT imaging, in patients with Chagas disease and left ventricular ejection fraction $(\mathrm{LVEF})>35 \%$. They divided the patients into three groups based on the severity of their VA. Group one included patients with sustained VA, group two included patients with at least two episodes of nonsustained VA, and group three included patients either with no VA or just one episode of nonsustained VA. The major findings of their study were as follows: First, there was no difference in the number of perfusion defects, severity of perfusion defects, and summed rest perfusion 
scores in the three groups. This observation suggests that the severity of ventricular arrhythmias in this patient population is independent of the size and severity of myocardial fibrosis as assessed by SPECT imaging. The second important finding of this study was the severity and extent of myocardial sympathetic denervation which was related to the burden of VA. Patients with sustained VA had the highest MIBG SPECT summed score and also the severity of the defects was higher in these patients. This result is not unique to CCC as similar findings were seen in the ADMIRE-HF and PARAPET trials which studied sympathetic denervation in patients with ischemic and nonischemic cardiomyopathy using planar MIBG and $\mathrm{C}^{11} \mathrm{PET}$ imaging, respectively. ${ }^{10,11}$ However, both these studies included patient with depressed LV function unlike the current study. Use of cardiac sympathetic denervation to predict arrhythmic risk in patient with normal or near normal LV function is certainly important in Chagas disease where traditional risk factors like LVEF may not be as useful. The third noteworthy finding, which authors do not stress as much, is the presence of different degrees of myocardial sympathetic denervation in patients with similar degree of myocardial fibrosis. The three groups of patients had similar resting myocardial perfusion imaging (MPI) summed score but different mIBG summed scores. This finding suggests that the mechanism of sympathetic neuronal damage may not be related to the microcirculation abnormalities responsible for perfusion defects. Why some patients with similar amount of fibrosis have larger amount of denervated myocardium is not known. Understanding what causes neuronal damage in Chagas disease may provide an opportunity for designing effective treatments.

The current study does have some obvious limitations like small sample size, single center experience, exclusion of patients with LVEF $<35 \%$ and can be considered as hypothesis generating. Nonetheless, it does contribute to our understanding of the disease process and reconfirms the presence of cardiac sympathetic denervation in CCC. It advances the possibility of risk stratifying patients with Chagas cardiomyopathy based on the extent and severity of their cardiac sympathetic denervation and intervening in those at highest risk. A conflicting finding in some case reports and small studies is the use of bilateral sympathectomy for treating patients with Chagas disease and refractory VA. ${ }^{12}$ This suggests that although partial sympathetic denervation is proarrhythmic, complete denervation may be protective. Another possibility is that rather than denervation, cardiac dysautonomia (mismatch between sympathetic and parasympathetic cardiac inputs) is more important for arrhythmogenesis. Currently, there are no reliable ways to image the cardiac parasympathetic nervous system; however, in future, studying the imbalance of sympathetic and parasympathetic cardiac inputs may provide new insights into the mechanism of VA not just in Chagas disease but also other cardiomyopathies.

\section{Disclosure}

The author declares no conflict of interest

\section{References}

1. Bestetti RB, Restini CB, Couto LB (2016) Carlos Chagas discoveries as a drop back to scientific construction of chronic Chagas heart disease. Arq Bras Cadiol.

2. Nunes MC, Dones W, Morillo CA, Encina JJ, Ribeiro AL. Chagas disease: An overview of clinical and epidemiological aspects. J Am Coll Cardiol 2013;62:767-76.

3. Elizari MV, Chiale PA. Cardiac arrhythmias in Chagas' heart disease. J Cardiovasc Electrophysiol 1993;4:596-608.

4. Koberle F. Chagas' disease and Chagas' syndromes: The pathology of American trypanosomiasis. Adv Parasitol 1968;6:63-116.

5. Amorim DS, Olsen EG. Assessment of heart neurons in dilated (congestive) cardiomyopathy. Br Heart J 1982;47:11-8.

6. Eckberg DL, Drabinsky M, Braunwald E. Defective cardiac parasympathetic control in patients with heart disease. N Engl J Med 1971. doi:10.1056/NEJM197110142851602.

7. Marin-Neto JA, Cunha-Neto E, Maciel BC, Simoes MV. Pathogenesis of chronic Chagas heart disease. Circulation 2007;115:1109-23.

8. Simoes MV, Pintya AO, Bromberg-Marin G, Sarabanda AV, Antloga CM, Pazin-Filho A, et al. Relation of regional sympathetic denervation and myocardial perfusion disturbance to wall motion impairment in Chagas' cardiomyopathy. Am J Cardiol 2000;86:975-81.

9. Kumar V, Chaterjee A. Ventricular arrhythmias and autonomic nervous system: evolving role of radionuclide imaging. $\mathrm{J}$ Nucl Cardiol 2016. doi:10.1007/s12350-016-0411-9.

10. Narula J, Gerson M, Thomas GS, Cerqueira MD, Jacobson AF. ${ }^{123}$ I-MIBG imaging for prediction of mortality and potentially fatal events in heart failure: The ADMIRE-HFX study. J Nucl Med 2015;56:1011-8.

11. Fallavollita JA, Jr Luisi Aj, Michalek SM, Valverde AM, deKemp RA, Haka MS, et al. Prediction of arrhythmic events with positron emission tomography: PAREPET study design and methods. Contemp Clin Trials 2006;27:374-88.

12. Saenz LC, Corrales FM, Bautista W, Traina M, Meymandi S, Rodriguez DA, et al. Cardiac sympathetic denervation for intractable ventricular arrhythmias in Chagas disease. Heart Rythm 2016. doi:10.1016/j.hrthm.2016.03.014. 